\title{
Optimal span length in high-speed transmission systems with hybrid Raman-erbium-doped fiber amplification
}

\author{
Juan Diego Ania-Castañón, Irina O. Nasieva, and Sergei K. Turitsyn \\ School of Engineering and Applied Science, Aston University, Birmingham B4 7ET, UK
}

Nicolas Brochier and Erwan Pincemin

France Telecom, Division R\&D, 2 Avenue Pierre Marzin, 22307 Lannion, France

Received August 5, 2004

\begin{abstract}
We show, using nonlinearity management, that the optimal performance in high-bit-rate dispersionmanaged fiber systems with hybrid amplification is achieved for a specific amplifier spacing that is different from the asymptotically vanishing length corresponding to ideally distributed amplification [Opt. Lett. 15, 1064 (1990)]. In particular, we prove the existence of a nontrivial optimal span length for 40-Gbit/s wavelength-division transmission systems with Raman-erbium-doped fiber amplification. Optimal amplifier lengths are obtained for several dispersion maps based on commonly used transmission fibers. (C) 2005 Optical Society of America

OCIS codes: $060.4510,060.4370,060.2320$.
\end{abstract}

During the late 1990s, erbium-doped fiber amplifiers (EDFAs) had a strong effect on the performance and design of optical networks, resulting in the deployment of a large number of high-capacity long-distance optical transmission systems. In this context, the optimization of the amplifier spacing allows one to control the amplified spontaneous emission (ASE) noise generated by the cascade of EDFAs. In fact, the finest amplification span length is one that facilitates the best trade-off between the low-cost requirements (a low number of amplifiers, ensuring satisfying cost efficiency) and stringent system performance constraints (a large number of amplifiers, ensuring good performance of the transmission system because of the reduction of accumulated ASE noise). Nonlinear effects also have a strong influence on overall system performance. In particular, long amplifier spans result in high input average powers (to maintain a good optical signal-to-noise ratio), leading in turn to an increase in the effects of nonlinearities. In these conditions the goal of transmission-system designers is to find the best balance between the requirements of a high optical signal-to-noise ratio (OSNR) and few nonlinear impairments. Yariv demonstrated ${ }^{1}$ that the highest OSNR of an ideal optically amplified system is obtained for a transmission system that produces a perfectly distributed amplification or asymptotically vanishing span length. This result is incompatible with the modern necessity for cheaper system design. Fortunately, the recent availability of reliable high-power laser pumps has made possible a comeback of distributed Raman amplification (DRA) in dense wavelength-division multiplexing (WDM) transmission systems. ${ }^{2-4}$ Compared with traditional lumped amplifier schemes, DRA significantly improves the link's OSNR. The margins released can then be used for extending the transmission distance and (or) decreasing the signal power injected into the fiber span (thus limiting the effects of nonlinearities). Combined with dispersion management and EDFA amplification, distributed Raman amplification can be used to better control the evolution of signal power inside the amplification spans, and thus nonlinear effects along the optical line, effectively performing nonlinearity management. ${ }^{5-7}$ The properties of the fibers used (Raman gain efficiency, attenuation, effective area, Rayleigh backscattering coefficient) and the design of the dispersion map have then to be taken into account when one is configuring the amplification scheme. $^{8}$

In this Letter we highlight new design possibilities for high-speed optical communications and present some examples in which optimal performances do not necessarily require shorter amplifier spacing. As we will show, the implementation of DRA and nonlinearity management permits a significant increase in the amplifier spacing without degrading the output OSNR or exacerbating nonlinearities. By using the approach recently reported in Refs. 5-7, we investigate the effects of amplifier span length on the optimal configuration of the amplification scheme in 40-Gbit/s WDM transmission systems based on hybrid Raman-EDFA amplification and standard single-mode fiber (SSMF)-dispersion-compensating fiber (DCF) and superlarge-area (SLA)-inverse dispersion fiber (IDF) dispersion mapping.

If we consider nonlinearities as always contributing to the degradation of the system's performance, the nonlinear phase shift (NPS) can be considered a good criterion with which to measure the effect of nonlinear impairments. ${ }^{5-7,9-11}$ One can then determine the optimal system configuration by performing a conditional minimization of the NPS at a fixed OSNR or, vice versa, a maximization of the OSNR at a fixed NPS. Although this conditional minimization can be performed analytically for simple cases, a numerical approach is usually required. We have performed numerical modeling of 40-Gbit/s WDM transmission systems based on hybrid Raman-EDFA amplification and various dispersion maps, using the average power equations for the Raman pump, signal, and noise, to find the optimal system parameters (the optimal gain split between the lumped and distributed 
amplifiers and the best amplifier spacing) that allow for minimization of the NPS at a fixed OSNR. The numerical approach ensures that all important effects, including double Rayleigh backscattering (DRS) noise and pump depletion, are accounted for. ${ }^{6,7}$

Although the method applied is general, we focus here on two basic configurations, depicted in Fig. 1. In both cases, a two-step dispersion map with hybrid Raman-EDFA amplification is considered, but the position of the backward Raman pump differs in each configuration. In configuration (a) (Fig. 1, top) the backward Raman pump is placed immediately after the section of positive dispersion fiber (Fiber 1) and an EDFA, with a typical noise figure of $4.5 \mathrm{~dB}$, is used for postamplification at the end of the span. In configuration (b) (Fig. 1, bottom) the backward Raman pump and the EDFA are located together at the end of the periodic transmission cell. The combined distributed-lumped gain compensates exactly for the total attenuation that is due to the transmission through the two fiber sections.

We consider WDM transmission at $40 \mathrm{Gbits} / \mathrm{s}$ with equally spaced channels (the channel spacing is $100 \mathrm{GHz}$ ) symmetrically distributed around a central 1550-nm wavelength. Two different dispersion maps are investigated for configuration (a): one based on SSMF with DCF and another one based on SLA fiber with IDF. ${ }^{12,13}$ For configuration (b), only the SLA-IDF option is considered. The features of the fibers used are summarized in Table 1 . The ratio $\eta$, defined as the quotient between the on-off Raman gain and the total on-off gain, both in decibels, characterizes the hybrid amplification scheme. The total length of the span is considered a variable. The ratio between positive and negative dispersion fiber section lengths is fixed for each fiber pair, to have zero accumulated dispersion. The total transmission distance is fixed to an arbitrary and sufficiently long $900 \mathrm{~km}$ of SSMF for the first fiber pair and of combined SLA-IDF for the second. The number of spans (always an integer) varies with the span length to maintain a fixed transmission distance.

Figure 2 displays the NPS in a contour plot versus length $L$ of the span and gain ratio $\eta$, for a fixed output OSNR of $22 \mathrm{~dB}$ (the bandwidth for the noise measurement is fixed at $100 \mathrm{GHz}$ ) in configuration (a) for both fiber pairs. We can observe that there is a clear optimal length for the amplification span (for which the NPS is minimal), which is $\sim 50 \mathrm{~km}$ (of SSMF) for SSMF-DCF and $\sim 90 \mathrm{~km}$ (60 km of SLA) for SLA-IDF. This optimal cell length can be understood as the one that allows us to find the best balance between the two following basic variants:
- The short-span regime, in which the signal is transmitted in quasi-lossless conditions, which results in large accumulated NPS even from relatively low input signal powers.

- The long-span regime, in which the long distance between amplifiers leads to increased ASE and DRS noise, forcing the input signal power to rise to maintain the output OSNR constant, thus exacerbating nonlinearities.

It can also be derived from Fig. 2 that the optimal $\eta$ varies slightly with the span length (when the span is longer than $20-30 \mathrm{~km}$ for the two basic maps considered here), becoming a bit lower for long amplification spans. This phenomenon can be explained by the growth of the Raman pump power and gain and, with it, of the contribution of DRS noise (owing to a worse distribution of the gain within the span) when the span length increases. To recover the best possible performance, then, a reduction of the Raman gain and an increase on the contribution of the lumped amplifier is required. For span lengths shorter than $20-30 \mathrm{~km}$ there is no clear optimal $\eta$ for the two cases considered here. For $100 \mathrm{~km}$ and longer SSMF or SLA-IDF, however, the determination of the optimal $\eta$ becomes crucial, as shown in Fig. 2.

It is also important to study the amount of dependence of the optimal span length that minimizes the nonlinear impairments on the targeted output OSNR. By varying the required output OSNR we demonstrate that the optimal span length is virtually independent of the OSNR constraint.

Finally, Fig. 3 shows the results obtained for configuration (b). The change in configuration has a clear effect on the optimal span length, which is reduced to $\sim 50 \mathrm{~km}$ of combined SLA-IDF compared with configuration (a), for which the optimum was $\sim 90 \mathrm{~km}$. For a low fixed output OSNR, the optimal amplifier configuration corresponds to values of $\eta$ close to 1 (full

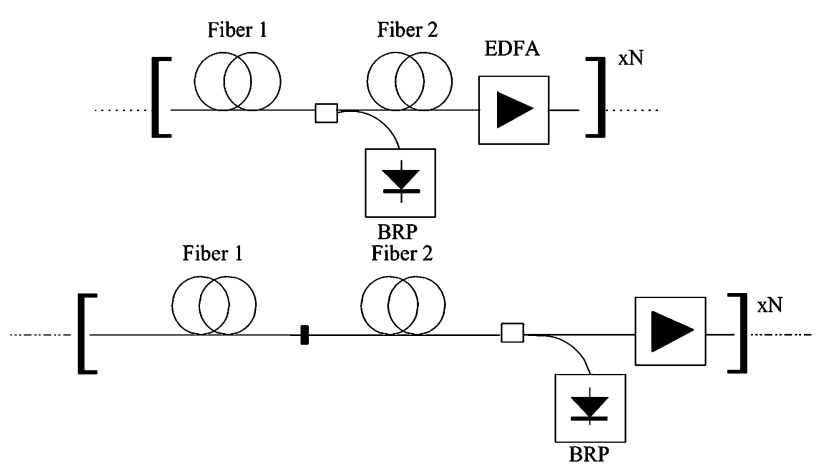

Fig. 1. System configurations considered. Top, configuration (a); bottom, configuration (b).

Table 1. Characteristics of the Fibers Used

\begin{tabular}{lcccccc}
\hline Fiber & Dispersion $[(\mathrm{ps} / \mathrm{nm}) / \mathrm{km}]$ & $\alpha_{1455}(\mathrm{~dB} / \mathrm{km})$ & $\alpha_{1550}(\mathrm{~dB} / \mathrm{km})$ & $A_{\text {eff }}\left(\mu \mathrm{m}^{2}\right)$ & $G\left(\mathrm{~W}^{-1} \mathrm{~km}^{-1}\right)$ & $\kappa\left(10^{-4} \mathrm{~km}^{-1}\right)$ \\
\hline SSMF & 17 & 0.257 & 0.2 & 80 & 0.39 & 0.64 \\
DCF & -100 & & 0.65 & 19 & & 7.1 \\
SLA & 20.2 & 0.234 & 0.188 & 106 & 0.29 & 0.4 \\
IDF & -40.4 & & 0.233 & 30 & & 1.6 \\
\hline
\end{tabular}



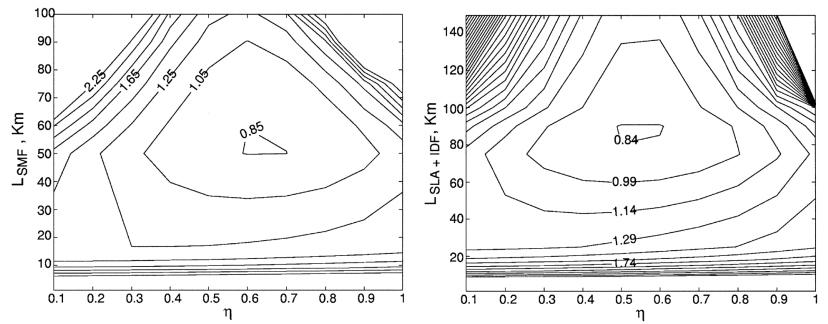

Fig. 2. NPS versus $L$ (in kilometers) and $\eta$ for a fixed OSNR of $22 \mathrm{~dB}$ after $900 \mathrm{~km}$ of total transmission through SSMF-DCF and SLA-IDF (right) with configuration (a).
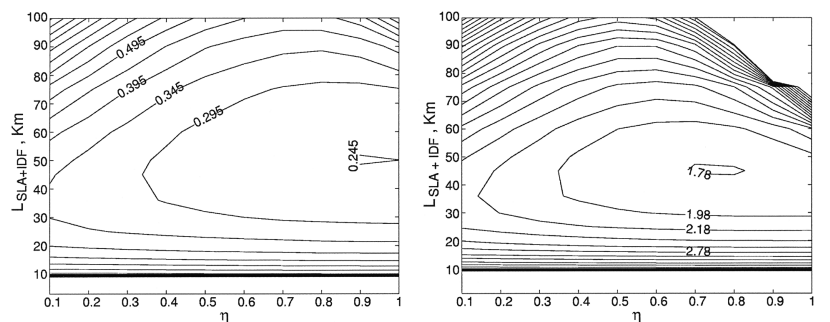

Fig. 3. NPS versus $L$ (in kilometers) and $\eta$ for fixed OSNRs of $22 \mathrm{~dB}$ (left) and $30 \mathrm{~dB}$ (right) after $900 \mathrm{~km}$ of total transmission through SLA-IDF with configuration (b).

Raman amplification), but, when the OSNR or the span length is increased, the optimal $\eta$ is reduced to counteract the effect of DRS noise (the optimal configuration then requires that there be greater participation from the EDFA to limit DRS noise). As happened with configuration (a), the increase in the output OSNR to $30 \mathrm{~dB}$ has a negligible effect on the optimal span length. Note that, in all cases shown, and even for the longest span lengths considered, the power required from the Raman pump to provide optimal Raman gain is below $1 \mathrm{~W}$, well within the possibilities of mode fiber lasers.

It is interesting to note that, for similar span lengths and the same SLA-IDF fiber base, configuration (b) accumulates less NPS than configuration (a) at equivalent noise levels. It seems, then, that the possibility of using DRA for the whole span, while at the same time being able to control DRS by increasing the contribution of the EDFA when high gains are required, gives configuration (b) greater flexibility. Such indeed does not have to be the case for other fiber combinations, but the low attenuation and relatively high effective core area of IDF makes it possible in this case to pump the negative dispersion fiber directly without too much exacerbation of DRS noise. Nevertheless, it is important to take into account that the differences between the two dispersion maps can be expected to affect the degree to which nonlinearities affect the signal and that of a lower accumulated NPS, although it is orientational, cannot be translated directly into a better bit-error-rate performance when systems based on different kinds of fiber are compared.

In conclusion, the existence of a nontrivial optimal amplifier spacing, matching modern requirements for cheap system design, has been demonstrated for 40-Gbit/s WDM dispersion-managed transmission systems that use a hybrid Raman-EDFA amplification scheme. A general method for determining the optimal span length, based on the concepts of noise and nonlinearity management, has been presented. Using this approach, we have shown the existence of this optimal amplifier spacing in 40-Gbit/s WDM transmission systems, considering various dispersion maps (using different fiber arrangements) and hybrid Raman-EDFA amplification schemes. The optimization results are nearly independent of the desired output OSNR; hence the optimal span length determined for each system through this method is applicable to a wide range of cases.

J.-D. Ania-Castañón's e-mail address is aniacajd@ aston.ac.uk.

\section{References}

1. A. Yariv, Opt. Lett. 15, 1064 (1990).

2. C. Rasmussen, T. Fjelde, J. Bennike, F. Liu, S. Dey, B. Mikkelsen, P. Mamyshev, P. Serbe, P. van der Wagt, Y. Akasaka, D. Harris, D. Gapontsev, V. Ivshin, and P. Reeves-Hall, in Optical Fiber Communication Conference $(\mathrm{OFC})$, Vols. 95A and 95B of OSA Trends in Optics and Photonics Series (Optical Society of America, Washington, D.C., 2003), paper PD18.

3. T. Tsuritani, K. Ishida, A. Agata, K. Shimomura, I. Morita, T. Tokura, H. Taga, T. Mizuochi, and N. Edagawa, in Optical Fiber Communication Conference (OFC), Vols. 95A and 95B of OSA Trends in Optics and Photonics Series (Optical Society of America, Washington, D.C., 2003), paper PD23.

4. M. Mehendale, M. Vasilyev, A. Kobyakov, and M. Williams, Electron. Lett. 38, 648 (2002).

5. S. K. Turitsyn, M. P. Fedoruk, V. K. Mezentsev, and E. G. Turitsyna, Electron. Lett. 39, 29 (2003).

6. I. O. Nasieva, J. D. Ana-Castañón, and S. K. Turitsyn, Electron. Lett. 39, 856 (2003).

7. J. D. Ania-Castañón, I. O. Nasieva, N. Kurukitkoson, S. K. Turitsyn, C. Borsier, and E. Pincemin, Opt. Commun. 233, 353 (2004).

8. E. Pincemin, D. Grot, C. Borsier, J. D. Ania-Castañón, and S. K. Turitsyn, IEEE Photon. Technol. Lett. 16, 2362 (2004).

9. V. E. Perlin and H. G. Winful, in Optical Fiber Communication Conference (OFC), Vol. 86 of OSA Trends in Optics and Photonics Series (Optical Society of America, Washington, D.C., 2002), paper WB1.

10. J.-C. Antona, S. Bigo, and J.-P. Faure, in Optical Fiber Communication Conference (OFC), Vol. 86 of OSA Trends in Optics and Photonics Series (Optical Society of America, Washington, D.C., 2002), paper WX5.

11. A. Kobyakov, M. Vasilyev, S. Tsuda, G. Giudice, and S. Ten, in 28th European Conference on Optical Communication (ECOC), Vol. 3 of Proceedings of ECOC 2002 (COM, Technical University of Denmark, Lyngby, Denmark, 2002), paper 1.13.

12. S. N. Knudsen, M. O. Pedersen, and L. Gruner-Nielsen, Electron. Lett. 36, 2067 (2000).

13. B. Zhu, S. N. Knudsen, L. E. Nelson, D. W. Peckham, M. O. Pedersen, and S. Stulz, Electron. Lett. 37, 1467 (2001). 